\title{
The dependence of the performance of the process of finishing vibration processing on the shape of the abrasive tool (granules)
}

\author{
Aleksandra Kozhukhova ${ }^{1,{ }^{*}}$, Larisa Tolmacheva ${ }^{1}$, Irina Dmitrieva ${ }^{1}$, Inessa Kalyakina ${ }^{1}$ \\ ${ }^{1}$ Polytechnic Institute (branch) of the Don State Technical University in Taganrog, 109a, Petrovskaya \\ str., 347904, Taganrog, Russia
}

\begin{abstract}
The article presents the results of experimental studies of the influence of polymer abrasive granules shape on the surface roughness formation and metal removal during vibrational finishing and hardening treatment (VFHT). As is known, surface roughness and the amount of metal removal are affected by a number of factors: binder material, material and granularity of the abrasive, technological lubricant-coolant medium (LCM) type, loading mass, initial roughness, etc. Processing was carried out for $120 \mathrm{~min}$ on a UVG-4x10 vibrating machine, with a working chamber volume of $10 \mathrm{dm}^{3}$. Metal removal Q (grams) and surface roughness $\mathrm{Ra}(\mu \mathrm{m})$ were measured using an $\mathrm{AD}-200$ analytical balance and an SJ-210 profilometer. The results presented show that polymer abrasive granules have high cutting ability, the intensity of which depends on the shape of the granules.
\end{abstract}

\section{Introduction}

The main task of modern machinery production is to ensure machine parts operational characteristics. The main operational characteristics include: strength, wear resistance, roughness, accuracy, etc. To achieve the specified characteristics, final treatment, or finishing is applied, which consists in removing burrs, blunting sharp edges from the surface, as well as forming of a radius or a bevel. Such treatment is required to fulfill the conditions of other technological operations, such as assembly.

Finishing operations can be performed manually or by mechanized methods. The complexity of manual finishing operations of parts obtained by milling is from 40 to $65 \%$ [1].

Such complexity in the conditions of mass production is unacceptable; therefore, to increase productivity, a number of traditional and non-traditional processing methods can be used. The traditional ones include blade cutting tools improvement. Non-traditional methods include mechanized refinement of parts by means of surface plastic deformation.

Vibrational-abrasive treatment is an effective method of processing metal parts with overall dimensions up to $300 \mathrm{~mm}$. Depending on the processing medium characteristics, vibroabrasive treatment is a mechanochemical or mechanical removal of metal particles,

*Corresponding author: sanadstu@mail.ru 
including burrs, with simultaneous plastic deformation and hardening of the surface layer [2]. The process is carried out in a container filled with a mixture of a working medium (abrasive granules) and treated parts. The container makes oscillatory movements, which leads to the circulation of the mixture [3]. This method has the advantage of producing radius edges and can be used to process parts of any configuration and complexity.

The treatment efficiency depends on its mode and duration, on the parts material properties, on the abrasive medium granularity, on the abrasive granules size, on the granules adhesive properties, on the properties of the cutting fluid and its volume [4].

Thus, one of the ways to increase vibroabrasive treatment productivity is a rational choice of abrasive granules parameters.

Abrasive granules in an oscillating container (Fig. 1) can be represented as a continuous medium (liquid), which provides for considering the processes resulting from the treatment from the point of view of the properties of a compressible liquid [5].

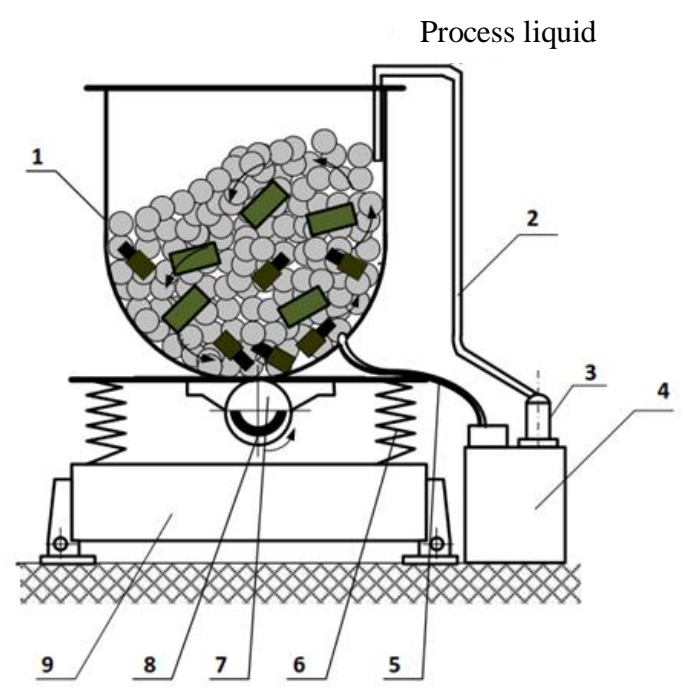

Fig. 1. UVG vibration machine schematic - 4x10: 1 - working chamber; 2 - LCM supply pipe; 3 pump; 4 - sedimentation tank; 5 - LCM outlet pipe; 6 - shock absorbers; 7 - vibration machine; 8 vibrator eccentric mass; 9 - base.

A single granule is a solid body of a certain shape, on the surface and in a cross section of which abrasive grains are located at a certain distance from each other [6].

For vibroabrasive treatment, specially made abrasive granules of a regular geometric shape are used; they include abrasive particles made of bundled grains or grinding powder, as well as production waste made from grinding wheels and ceramic-mineral breakage [7].

However, there is no widespread production of abrasive granules to meet the needs of industry in the Russian Federation, and what is produced in terms of cost and quality is inferior to imported counterparts.

The prospect of increasing the productivity of vibroabrasive treatment is to improve the quality of surfaces' treatment without changing treatment modes, machine power, energy consumption of the process, etc. These problems can be solved through the use of artificial abrasive materials when creating granules with the desired properties: increased durability and resistance to chemical influences. Such granules will have predetermined parameters: cutting properties, graininess, uniformity of structure and appearance. All this will make it possible to control processing parameters in a wide range, to optimize the process, and therefore to ensure its efficiency.

M.A. Kalmykov [8] conducted theoretical and experimental studies to evaluate the 
effectiveness of the use of abrasive granules of various shapes. The author revealed that granules shaped as a tetrahedron and a trihedral prism have the greatest cutting properties. Treatment in tetrahedral granules provides for the process productivity increase by $10 \ldots$ $80 \%$. This is explained by the fact that the flat faces of the tetrahedral shape have the largest contact area with the treated surface. The results of experimental studies into the effectiveness of using abrasive granules of various shapes, carried out by the same author [8], showed that tetrahedral granules have the best indicators for achieving the required surface roughness.

The author also studied cubic abrasive granules, which proved to have surface contact area not much different from that of a pyramid or a prism. However, according to the experimental studies, the removal of metal from the surfaces of samples of various geometric shapes by cubic granules is $30 \%$ less compared to tetrahedral and trihedral granules.

In [8], the author claims that the removal of metal during flat surfaces treatment in tetrahedral and prismatic granules is approximately the same; it is $35-45 \%$ more effective compared to granules of conical and cylindrical forms, and approximately twice as effective as in granules in the form of a ball. Based on the above results, it is difficult to precisely assess the effectiveness of an abrasive tool of one or another shape, and, therefore, this issue needs further consideration.

In [9], it was determined that the removal of metal occurs due to pinpoint impacts of the granule on the surface of the treated parts. Adhering to this theory, if the treatment is to be carried out on points we should choose an abrasive tool in the shape of a ball; in other words, the author considers the case when vibrational treatment takes place without the circulatory movement of the entire mass of the load, because in the presence of the latter, the pinpoint impact becomes linear.

However, it was established in [10] that $75 \%$ of all impacts on the treated surface during vibrational treatment are oblique, and, consequently, the point contact becomes linear and the linear contact becomes plane contact.

Thus, literature review shows that issues related to the influence of abrasive tool parameters on the treatment productivity and the wear resistance of granules were considered differentially and need to be clarified.

\section{Materials and Methods}

Preliminary analysis has shown that it is necessary to study the shape of the granules and its effect on the main parameters of treatment. The research methodology assumes that the only variable parameter is the shape of the granules; all other parameters are assumed to be the same, namely:

1. Granules are made on a polymer bond PMMA + MMA, using normal electrocorundum with grain size of 1000 microns (EN-10) as a filler. This choice is due to the fact that the granules on a polymer bond have high wear resistance, good cutting properties and can take any shape, i.e. can be used for processing parts of various configurations [11].

2. The concentration of abrasive is $50-60 \%$, which is optimal according to previous studies.

These abrasive granules have the following composition [12]:

- abrasive : up to 51\% recommended (grain size can vary from 6 to 100 microns);

- polymer: beaded polymethylmethacrylate PMMA - 24\%;

- monomer: liquid methyl methacrylate MMA - 24\%;

- hardener : dimethylaniline - up to $1 \%$.

Abrasive granules shaped as cones, pyramids with a square at the base (P4); pyramids 
with a non-convex hexagon (P6) at the base, and two-sided pyramids, in the cross section of which there is a non-convex hexagon (P12) (Fig. 2) were produced and studied from the point of view of productivity and wear resistance.

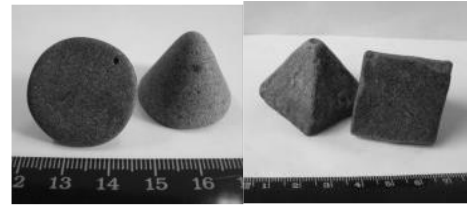

a)

b)

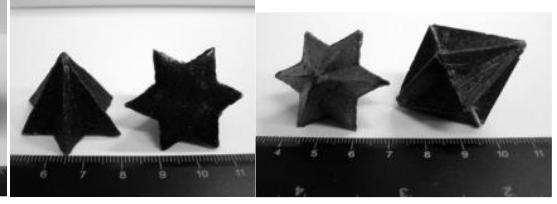

c)

d)

Fig. 2. Test abrasive granules: a) cones; b) P4; c) P6; d) P12.

Treatment was carried out for $120 \mathrm{~min}$ on a UVG-4x10 vibrating machine, with a working chamber volume of $10 \mathrm{dm}^{3}$. Metal removal Q (grams) and surface roughness $\mathrm{Ra}$ $(\mu \mathrm{m})$ were measured using an AD-200 analytical balance and an SJ-210 profilometer.

\section{Results}

To assess the vibrational treatment quality indicators, the surfaces of the studied samples were analyzed, which condition before and after processing is shown in Fig. 3-4. For experimental research cylindrical and flat brass samples with sharp edges, traces of a blade tool, and rust on surfaces were used.

A visual inspection showed that after vibrational treatment, all of the samples had a matte uniform color; sharp edges were rounded, no traces of the tool remaining after pretreatments were observed. The roughness of the surface layer of the samples after processing changed from $\mathrm{Ra}=4.1-3.4 \mu \mathrm{m}$ to $\mathrm{Ra}=1.3-1.15 \mu \mathrm{m}$ (Table 1 ), which is more than two classes higher.

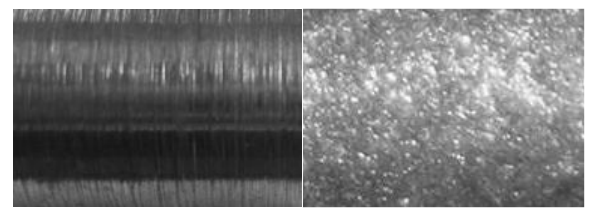

a)

b)

Fig. 3. The surface of cylindrical brass samples: a) before vibrational treatment; b) after vibrational treatment.

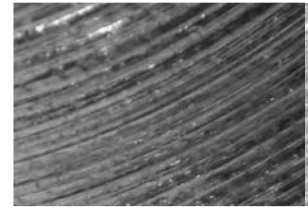

a)

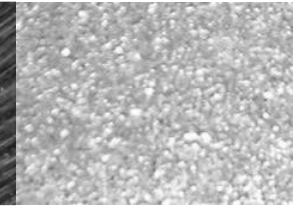

b)

Fig. 4. The surface of flat brass samples: a) before vibrational treatment; b) after vibrational treatment.

It should be noted that the same surface roughness results were achieved in different time intervals. Therefore, to reduce operating time and increase productivity of vibrational treatment of parts, regardless of their material and initial roughness, the use of P12-shaped abrasive granules is the most rational. Possessing high cutting abilities, P12 granules wear evenly during operation, while maintaining their original shape, which ensures constant high productivity of the tool used throughout the entire period of its operation. 
Table 1. The results of experimental studies of the processed samples' roughness.

\begin{tabular}{|c|c|c|c|c|c|c|}
\hline \multirow{2}{*}{$\begin{array}{c}\text { Sample type, } \\
\text { dimensions and } \\
\text { material }\end{array}$} & \multicolumn{2}{|c|}{$\begin{array}{c}\text { Surface roughness, Ra, } \\
\boldsymbol{\mu m}\end{array}$} & \multicolumn{4}{c|}{ Technological operation time, min } \\
\cline { 2 - 6 } & $\begin{array}{c}\text { Before } \\
\text { treatment }\end{array}$ & $\begin{array}{c}\text { After } \\
\text { treatment }\end{array}$ & Cone & $\begin{array}{c}\text { Pyramid } \\
(\mathbf{P 4})\end{array}$ & $\begin{array}{c}\text { Pyramid } \\
(\mathbf{P 6})\end{array}$ & $\begin{array}{c}\text { Pyramid } \\
(\mathbf{P 1 2})\end{array}$ \\
\cline { 4 - 7 } \\
$\begin{array}{c}\text { Solid cylinders, } \\
\varnothing 25 \times 10 \mathrm{~mm} \text {, brass LS } \\
59-1 \mathrm{~L}\end{array}$ & $3.4 \ldots 3.5$ & $1.15 \ldots 1.3$ & 60 & 55 & 50 & 35 \\
\hline $\begin{array}{c}\text { Parallelepipeds, } \\
20 \times 10 \times 10 \mathrm{~mm}, \text { brass } \\
59-1 \mathrm{~L}\end{array}$ & $3.5 \ldots 4.1$ & $1.18 \ldots 1.3$ & 60 & 50 & 45 & 35 \\
\hline
\end{tabular}

\section{Discussion}

Vibrational treatment contributes to the crushing of roughness peaks, to the increase in the profile reference length, increase in the radii of the protrusions' peaks, which leads to an increase in the contact area of mating surfaces and contributes to improving of the operational properties of products [13].

The shape of the abrasive granule, according to experimental studies, affects not only the performance of abrasive granules, but also on their mobility in the vibration field [14]. Studies have shown that the circulating velocity of the medium consisting of granules of various shapes changes on average by $25-30 \%$ compared with traditional cones.

These studies were carried out by graphical analysis of the video storyboards made on an industrial machine model UVG-4 $\times 10$, where a metal wall was replaced with a transparent one. The studies have shown that highly effective abrasive granules of different shapes (but with the same binding and abrasive components) under the influence of the same vibrations move with different speeds, both in different zones and medium, and, respectively, with different stability; they have different mobility and volume expansion. In the studied granules, the velocity spread is $21-37 \%$, which coincides with the results of [15-20].

\section{Conclusions}

To conclude: with the same treatment modes, the given roughness parameters in the shortest treatment time are achieved with granules shaped as two-sided pyramid, in the cross section of which there is a non-convex hexagon.

As a result, it can be noted that the use of a high-performance P12-shaped abrasive tool provides for the necessary technological result (in particular when processing complex configuration parts), and reduce the operational time by $40 \%$.

\section{References}

1. K. Hamouda, H. Bournine, H.E. Amrou, M.A. Tamarkin, A.P. Babichev, D. Saidi, Materials Science 52, 216-221 (2016) doi: 10.1007/s11003-016-9946-9

2. M.A. Tamarkin, A.N. Isaev, E.V. Murugova, V.I. Butenko, MATEC Web Of Conferences (2018) doi: 10.1051/matecconf/201822601025

3. M.A. Tamarkin, A.A. Tikhonov, E.E. Tishchenko, Russian Engineering Research 34, 175-177 (2014) doi: 10.3103/S1068798X14030150

4. M.A. Tamarkin, E.E. Tishchenko, V.P. Fedorov, IOP Conference Series: Materials 
Science and Engineering, 012169 (2016) doi: 10.1088/1757-899X/124/1/012169

5. A.P. Babichev, P.D. Motrenko, S.A. Kostenkov, O.A. Rozhnenko, M.A. Tamarkin, V.M. Shumyacher, Tool support of part treatment processes in granular environments (Don State Technical University, Rostov-on-Don, 2011)

6. M.A. Tamarkin, E.E. Tishchenko, O.A. Rozhnenko, Russian Engineering Research 33, 302-305 (2013) DOI: 10.3103/S1068798X1305016X

7. M.A. Tamarkin, E.E. Tishchenko, A.S. Shvedova, Russian Engineering Research 38(9), 726-727 (2018) DOI: 10.3103/S1068798X18090277

8. M.A. Kalmykov, T.A. Shumakova, I.M. Levinskaya, Vibration in technology Vinnitsa 3(55), 69-72 (2009)

9. P.L. Nosko, M.O. Kalmikov, A.P. Nikolaenko, L.M. Lubenska, Application of vibration processing to improve product quality: monograph (Knowledge Publishing House, Lugansk, 2009)

10. M.A. Tamarkin, E.E. Tishchenko, D.V. Kazakov, A.G. Isaev, Russian Engineering Research 37(4), 326-329 (2017) DOI: 10.3103/S1068798X17040219

11. A.V. Kozhukhova, A.S. Ponomarenko, V.A. Timofeev, R.S. Karpenko, A.N. Tokarev, Collection of Selected Articles on the Materials of Scientific Conferences of the National Research Institute National Development, 42-45 (2019)

12. A.V. Kozhukhova, L.V. Tolmacheva, T.V. Novoselova, Collected papers of the XII International Scientific and Practical Conference within the framework of the XXII Agroindustrial Forum of the South of Russia and the exhibition "Interagromash", 417420 (2019)

13. M.A. Tamarkin, E.E. Tishchenko, V.G. Lebedenko, Russian Engineering Research 30(2), 144-148 (2010) DOI: 10.3103/S1068798X10020115

14. V.V. Nepomnyashchii, T.V. Mosina, S.M. Voloshchenko, K.A. Gogaev, M.G. Askerov, Refractories and Industrial Ceramics 55(3), 188-190 (2014) DOI: 10.1007/s11148-014-9687-y

15. Y.Y. Krupenya, M.A. Boyko, A.P. Shishkina, MATEC Web of Conferences, 01026 (2018) DOI: 10.1051/matecconf/201822601026

16. V.A. Lebedev, A.P. Shishkina, I.V. Davydova, A.V. Morozova, IOP Conference Series: Materials Science and Engineering Processing Equipment, Mechanical Engineering Processes and Metals Treatment, 042061 (2018) DOI: 10.1088/1757899X/327/4/042061

17. A.A. Elakova, A.P. Abyzov, V.B. Stupko, International Journal of Pharmacy and Technology 8(3), 14815-14821 (2016)

18. A.A. Yelakova, A.P. Abyzov, V.B. Stupko, International Journal of Applied Engineering Research 10(24), $44662-44667$ (2015)

19. A.A. Elakova, A.P. Abyzov, V.B. Stupko, International Journal of Pharmacy and Technology 8(4), 24447-24457 (2016)

20. V. Strutynsky, V. Symonyuk, Technological complexes 2(8), 137-143 (2013) 